\title{
ACOUSTIC RECTIFICATION IN DISPERSIVE MEDIA
}

\author{
John H. Cantrell \\ NASA Langley Research Center, Mail Stop 231, Hampton, VA 23681, USA
}

\begin{abstract}
It is shown that the shapes of acoustic radiation-induced static strain and displacement pulses (rectified acoustic pulses) are defined locally by the energy density of the generating waveform. Dispersive properties are introduced analytically by assuming that the rectified pulses are functionally dependent on a phase factor that includes both dispersive and nonlinear terms. The dispersion causes an evolutionary change in the shape of the energy density profile that leads to the generation of solitons experimentally observed in fused silica.
\end{abstract}

Keywords: Radiation-Induced Static Pulses, Dispersive Media, Solitons

PACS: $62.65 .+\mathrm{k}, 43.25 . \mathrm{Dc}, 43.25 . \mathrm{Qp}$, 43.25.Rq

\section{INTRODUCTION}

The propagation of a sinusoidal acoustic wave through a nonlinear medium is known to generate not only harmonics of the original waveform but also a static strain (acoustic rectification or "dc shift") associated with the propagating wave. Using an approach based on the virial theorem, Cantrell [1,2] showed that the static strain generated by an acoustic toneburst (gated sinusoidal wave) is dependent on the energy density of the waveform. He considered that the energy dependence is local and predicted that the derived static displacement pulse would propagate as a right-triangular pulse as a direct result of the locality. Experimental confirmation of the local energy dependence of static displacement pulses was reported by Yost and Cantrell [3] and Cantrell et al. [4] for monocrystals of silicon for wave propagation along the pure mode propagation directions. Yost and Cantrell [3] also reported results for fused silica - a dispersive material with a negative nonlinearity parameter. The pronounced spreading and variations in amplitude of the displacement pulse profile observed in fused silica, however, were not predicted by the model. We develop here a model of static pulses in dispersive materials that does predict the spreading and amplitude variation of the displacement pulse profile as the consequence of the generation of solitary waveforms (solitons) of the acoustic energy density. 


\section{ACOUSTIC RECTIFICATION IN NONDISPERIVE MEDIA}

We consider the propagation of an elastic wave in a lossless, non-dispersive, semi-infinite medium of arbitrary crystalline symmetry. The nonlinear equation of motion along an arbitrary propagation direction may be transformed into the form ${ }^{4}$ $\left(\partial^{2} u / \partial t^{2}\right)=c_{\varepsilon}^{2}\left[1-\beta_{\varepsilon}(\partial u / \partial a)\right]\left(\partial^{2} u / \partial a^{2}\right)$, where $\mathrm{t}$ is time, "a" is the coordinate along the wave propagation direction, $\mathrm{u}$ is the particle displacement, $\mathrm{c}_{\varepsilon}$ is the wave phase velocity where $\varepsilon$ is a mode index associated with given directions of wave propagation and polarization, and $\beta_{\varepsilon}$ is the modal nonlinearity parameter. The Earnshaw particle velocity $\partial \mathrm{u}_{\varepsilon} / \partial \mathrm{t}$ solution to the nonlinear wave equation for each mode $\varepsilon$ is [5]

$$
\partial u_{\varepsilon} / \partial t=A_{\varepsilon} \sin \left(\omega t-k_{\varepsilon} a+\phi\right), \quad \phi=k_{\varepsilon} a\left(\beta_{\varepsilon} / 2 c_{\varepsilon}\right)\left(\partial u_{\varepsilon} / \partial t\right)
$$

where $A_{\varepsilon}$ is the amplitude of the waveform, $\omega$ is the angular frequency, $k_{\varepsilon}=\omega / \mathrm{c}_{\varepsilon}$, and $\phi$ is a phase modulation factor, containing the nonlinearity parameter, that leads to wave distortion. For periodic waveforms of constant amplitude the acoustic strain rectification, or radiation-induced static strain $\left\langle\partial u_{\delta} \partial \mathrm{a}\right\rangle$, is conveniently obtained by time-averaging the displacement gradient $\partial u_{d} \partial a$ (the angular brackets denote time-averaging). The strain rectification (static strain) is related to the time-averaged particle velocity $\left\langle\partial u_{\delta} \partial \partial t\right\rangle$, hence time-averaged energy density $\langle E\rangle$, as $[1,2]$

$$
\left\langle\partial u_{\varepsilon} / \partial a\right\rangle=\left(\beta_{\varepsilon} / 4 c_{\varepsilon}^{2}\right)\left\langle\left(\partial u_{\varepsilon} / \partial t\right)^{2}\right\rangle=\left(\beta_{\varepsilon} / 4 \mu_{\varepsilon}\right)\langle E\rangle
$$

where $\mu_{\varepsilon}$ is the modal second-order Huang coefficient. The last equality results from the relations $E=\rho_{0}\left(\partial u_{\varepsilon} \partial t\right)^{2}$, where $\rho_{0}$ is the mass density of the propagation medium, and $c_{\varepsilon}^{2}$ $=\mu_{d} \rho_{0}$.

It is clear from Eq.(2) that the static strain is defined by the time-averaged energy density $\left\langle E>\right.$ of the propagating wave. The constants $\beta_{\varepsilon}$ and $\mu_{\varepsilon}$ serve as scaling factors for the static strain. Since the energy density for a toneburst in a non-dispersive, lossless medium propagates as a stationary waveform, the static strain must be local and therefore exists only in that region of space for which $\langle E\rangle$ is non-zero. Thus, from Eq.(1) the time-averaged energy density $\langle E\rangle=(1 / 2) \rho_{0} A_{\varepsilon}^{2}$ and the shape of the static strain pulse generated by a toneburst must be rectangular with height proportional to $A_{\mathcal{E}}^{2}$. Accordingly, the static displacement is obtained as the spatial integral of the rectangular static strain pulse only over the non-zero values of $\langle E\rangle$, or energy per unit mass $A_{\varepsilon}{ }^{2}$, commensurate with the stationary wave nature of the propagating energy density (or energy per unit mass). The locality both of the static strain and displacement pulses means that, in contrast to harmonic generation, the static displacement does not increase linearly with total distance of wave propagation, but rather increases linearly only over the spatial extent of the non-zero energy density or energy per unit mass. Hence, for a toneburst of length $L$ the static displacement pulse propagates in the shape of a righttriangular pulse with the peak value $\left\langle u_{\varepsilon}\right\rangle$ obtained from Eqs.(1) and (2) as 

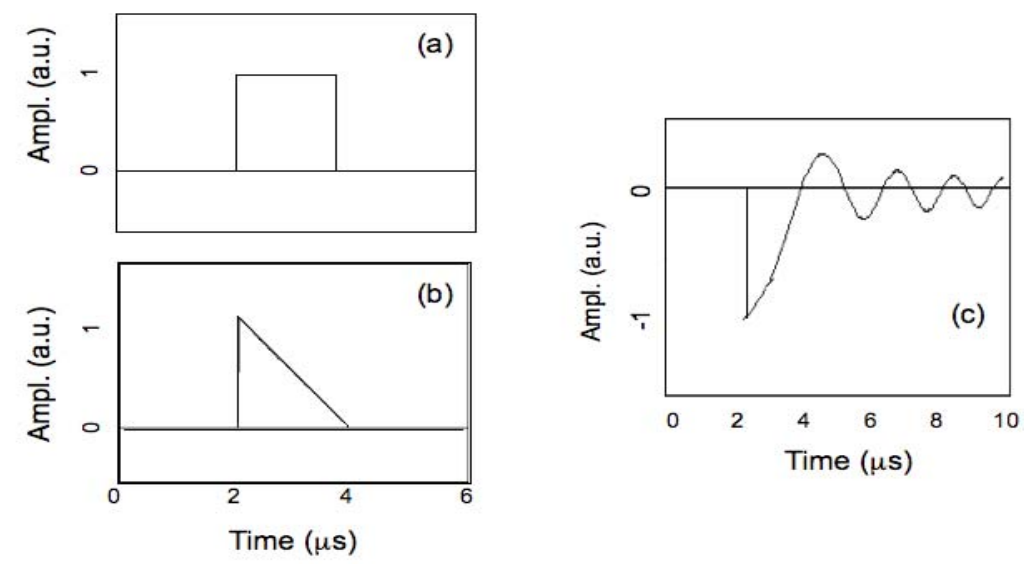

FIGURE 1. Rectified acoustic pulses generated by a $2 \mu$ s toneburst: (a) Static strain pulse in nondispersive media. (b) Static displacement pulse in non-dispersive media. (c) Static displacement pulse profile (soliton plus oscillatory tail) in dispersive media.

$$
\left\langle u_{\varepsilon}\right\rangle=(1 / 8) \beta_{\varepsilon} k_{\varepsilon}^{2} A_{\varepsilon}^{2} L
$$

Fig.1a shows a typical rectangular static strain pulse generated by a $2 \mu$ s toneburst as given by Eq.(2). Fig.lb shows a typical right-triangular static displacement pulse generated by a $2 \mu$ s toneburst as given by Eq.(3). The perspective in Fig. 1 is the signal as seen by the receiving transducer. Yost and Cantrell [3] obtained experimental confirmation of the right-triangular shape of the static displacement pulse in a silicon monocrystal by substituting a right-triangular pulse from a function generator into the detection electronics of the experimental set-up and comparing the output signal with that generated from the acoustic wave. The overlap of the two output signals indicated that the static displacement was indeed right-triangular in shape. Calculations of the nonlinearity parameters from measurements of the slopes of the static displacement pulses along the pure mode propagation directions in single crystal Si gave $\beta_{\varepsilon}$ values that are in agreement with nonlinearity parameter measurements obtained independently from acoustic harmonic generation measurements [4]. Such agreement provides strong evidence of the right triangular shape of the static displacement pulses.

\section{ACOUSTIC RECTIFICATION IN DISPERSIVE MEDIA}

Further evidence that the static strain and displacement pulses are defined locally by the energy density of the propagating wave is obtained from a consideration of the static strain and displacement pulses in vitreous silica - a dispersive material with a negative nonlinearity parameter. The dispersion can be accounted by generalizing the phase factor $\phi$ in Eq.(1) to include dispersive as well as nonlinear components. We 
assume that rectified pulses are generated by the toneburst in accordance with Eq.(2), where $\langle E\rangle=(1 / 2) \rho_{0} A_{\varepsilon}^{2}$, to yield $\langle\partial u / \partial a\rangle=\left(\beta / 8 c^{2}\right) A_{D}^{2}$, where we have dropped the modal subscripts and have replaced the energy per unit mass $A^{2}$ by $A_{\mathrm{D}}{ }^{2}$. For dispersive media the energy per unit mass $A_{\mathrm{D}}{ }^{2}$ is no longer assumed to be constant but rather is assumed to evolve with propagation distance as $A_{\mathrm{D}}{ }^{2}=A_{\mathrm{D}}{ }^{2}\left[k_{0} a-\omega_{0} t+\phi(a, t)\right]$. At $a=0$, we assume $A_{\mathrm{R}}{ }^{2}=A^{2}$, the initial energy per unit mass of the toneburst launched into the material. The parameters $\omega_{0}$ and $k_{0}$ are associated with the toneburst and represent values of the parameters in the linear approximation.

We assume that the nonlinear dispersion relation depends on $A_{\mathrm{D}}$ and the wave number $k$ as

$$
\omega=\omega\left(k, A_{D}^{2}\right)=\omega_{0}+c_{0}\left(k-k_{0}\right)+(1 / 2) c_{0}^{\prime}\left(k-k_{0}\right)^{2}+\Gamma A_{D}^{2}+\cdots,
$$

where a Taylor series expansion with respect to $k_{0}$ and $A_{\mathrm{D}}{ }^{2}=0$ is used to obtain the last equality in Eq.(4). The $c_{0}, c^{\prime}{ }_{0}$, and $\Gamma$ are expansion coefficients. We consider $\omega$ and $k$ to be dependent on time and propagation distance, and impose the geometrical optics postulate that $\omega(a, t)=-(\partial \theta / \partial t)=\omega_{0}-(\partial \phi / \partial t)$ and $k(a, t)=(\partial \theta / \partial a)=k_{0}+(\partial \phi / \partial a)$, where $\theta(a, t)=k_{0} a-\omega_{0} t+\phi(a, t)$. Substituting the expressions for $\omega(a, t)$ and $k(a, t)$ into Eq.(4) and adding a spatial second derivative term to account for dispersion [6], we obtain

$$
(\partial \phi / \partial t)+c_{0}(\partial \phi / \partial a)+(1 / 2) c_{0}^{\prime}(\partial \phi / \partial a)^{2}+\Gamma A_{D}^{2}+\left(c_{0}^{\prime} / 2 A_{D}\right)\left(\partial^{2} A_{D} / \partial a^{2}\right)=0
$$

Transforming to a set of coordinates given by $\xi=a-c_{0} t$ and $\tau=c_{0}^{\prime} t$, we re-write Eq.(5) in the form

$$
(\partial \phi / \partial \tau)+(1 / 2) c_{0}^{\prime}(\partial \phi / \partial \xi)^{2}+\left(\Gamma / c_{0}^{\prime}\right) A_{D}^{2}+\left(1 / 2 A_{D}\right)\left(\partial^{2} A_{D} / \partial \xi^{2}\right)=0
$$

Since we are interested in obtaining the propagation properties of the energy per unit mass $A_{D}{ }^{2}=E / \rho_{0}$, it is necessary to invoke an second equaton involving both $A_{\mathrm{D}}{ }^{2}$ and $(\partial \phi / \partial \xi)$. We thus invoke the conservation of energy expression

$$
\partial A_{D}^{2} / \partial t+\partial\left(c_{g} A_{D}^{2}\right) / \partial a=0
$$

where from Eq.(4) $c_{g}=\partial \omega / \partial k=c_{0}+(\partial \phi / \partial a)$ is the energy propagation velocity (group velocity) in the linear approximation. Again transforming to the set of coordinates given by $\xi=a-c_{0} t$ and $\tau=c_{0}^{\prime} t$, we re-write Eq.(7) in the form

$$
\partial A_{D}^{2} / \partial \tau+(\partial / \partial \xi)\left[A_{D}^{2}(\partial \phi / \partial \xi)\right]=0
$$

Assuming that both $A_{\mathrm{D}}{ }^{2}$ and $(\partial \phi / \partial \xi)$ have stationary solutions to Eqs.(6) and (8) of the form $f=f(\xi-V \tau)=f(z)$ where $z=(\xi-V \tau)$, we obtain from Eq.(8) 


$$
(\partial / \partial z)\left[V A_{D}^{2}-(\partial \phi / \partial \xi) A_{D}^{2}\right]=0 .
$$

We integrate Eq.(9) and set the integration constant to $V A^{2}$ where $A^{2}$ is the energy per unit mass of the toneburst at $a=0$. Solving the resulting expression for $(\partial \phi / \partial \xi)$, we obtain

$$
\partial \phi / \partial \xi=V\left(1+\frac{A^{2}}{A_{D}^{2}}\right)
$$

Following Karpman [7], we now assume that $\phi=-\mu_{0}^{2} \tau+\phi_{1}(\xi-V \tau)$, substitute the expression into Eq.(6), and use Eq.(10) to obtain

$$
-\mu_{0}^{2}-\frac{1}{2} V^{2}\left(1-A_{0}^{4} A_{D}^{-4}\right)+\left(\Gamma / c_{0}^{\prime}\right) A_{D}^{2}+\left(1 / 2 A_{D}\right)\left(\partial^{2} A_{D} / \partial \xi^{2}\right)=0 .
$$

The stationary solution to Eq.(11) has the form of a solitary wave (soliton) given by [7]

$$
A_{D}^{2}=A_{0}^{2}+A_{S}^{2} \sec h^{2} \sqrt{\Gamma / c_{0}^{\prime}} A_{S}(\xi-V \tau)
$$

where

$$
V=\left(\Gamma / c_{0}^{\prime}\right)^{1 / 2}\left(A_{0}^{2}+A_{S}^{2}\right)^{1 / 2}
$$

and $A_{S}^{2}$ is the amplitude of the soliton.

Strictly, Eq.(12) is the general stationary solution for a perturbation imposed on a continuous carrier wave of amplitude $A_{0}$ and is valid for an arbitrary amplitude of perturbation [7]. If we assume that the perturbation (the toneburst) is large compared to the carrier amplitude, then in the limit where the carrier amplitude becomes arbitrarily small we obtain

$$
A_{D}^{2}=A_{S}^{2} \sec h^{2} \sqrt{\Gamma / c_{0}^{\prime}} A_{S}(\xi-V \tau)
$$

where $V=\left(\Gamma / c_{0}^{\prime}\right)^{1 / 2} A_{S}$. The number of solitons generated, each having different amplitudes $A_{s}^{2}$, depends on the length and amplitude of the initial perturbation. For a toneburst of amplitude $A$ the initial rectification is a rectangular static pulse of amplitude $A^{2}$. The complete solution also includes an oscillatory tail wave packet similar in form to that generated by the Airy function. The static strain $\langle\partial u / \partial a\rangle$ is obtained directly from Eqs.(2) and (14) as $\langle\partial u / \partial a\rangle=\langle\partial u / \partial \xi\rangle=(\beta / 4 \mu)\langle E\rangle$ where $\langle E\rangle=$ $(1 / 2) \rho_{0} A_{\mathrm{D}}^{2}$.

Fig.1c shows the static displacement pulse generated by a $2 \mu$ s toneburst obtained by integrating Eq.(14) and the oscillatory tail with respect to $\xi$. The soliton is detected 
first, since it has the greater velocity, and is followed by the slower oscillatory tail. It is important to note that the rectified acoustic pulses are generated by the toneburst at $a=0$. The dispersive and nonlinear properties of the rectified pulses are governed by the values of the expansion coefficients in the dispersion relation, Eq.(4), evaluated at the values of $k_{0}$ and $\omega_{0}$ associated with the toneburst. Thus, it should not be expected that launching a pulse in the material directly from a pulse generator would necessarily generate solitons, since the values of $k_{0}$ and $\omega_{0}$ used to evaluate the coefficients in Eq.(4) from such a pulse would not be the same as that of the rectified pulses generated by the toneburst.

\section{MEASUREMENT OF THE ACOUSTIC NONLINEARITY PARAMETER IN DISPERSIVE MEDIA}

Fig.1c shows the calculated static displacement profile, as observed by the receiving wave detector, resulting from a single soliton with an oscillatory tail generated by a $2 \mu$ s tone-burst in a dispersive medium with $\beta<0$. Note that since $\beta<0$, the displacement profile is inverted relative to that of Figs. $1 \mathrm{a}$ and $1 \mathrm{~b}$ where $\beta>0$. The pulse profile of Fig. 1c is also considerably longer than the toneburst duration and shows large variations in displacement amplitude. Both the spreading and the variations in amplitude of the static displacement pulse have been observed experimentally [3]. This observation provides further evidence that the static strain and displacement pulses are defined locally by the energy density of the waveform.

We consider the implications of the static pulse profile to the measurement of the acoustic nonlinearity parameter in dispersive media. The initial portion of the static displacement pulse in Fig.1c results from the soliton contribution to the profile. The slope of the pulse profile following the first peak is given by $\langle\partial u / \partial a\rangle=(\beta / 4 \mu)<E>$. At the half-peak height of that slope, where $\xi=V \tau$, the average energy density is evaluated from Eq.(9) to be $\left\langle E>=(1 / 2) \rho_{0} A{ }_{\mathrm{s}}{ }^{2}\right.$. Generally, $A_{\mathrm{s}}{ }^{2}$ is not equal to the square of the amplitude of the tone-burst. However, for a single soliton generated by a toneburst of appropriate length and amplitude it can be approximately so. If we assume that $A_{\mathrm{s}}{ }^{2}$ is very nearly equal to the square of the tone-burst amplitude, then measurement of the slope at $\xi=V \tau$ allows a direct, although approximate, calculation of $\beta$. Yost and Cantrell [3] performed such a calculation of $\beta$ for Suprasil W1 vitreous silica, fortuitously at $\xi=V \tau$, without benefit of the present analysis. Their calculated value of -12.7 for $\beta$ is in quite good agreement with the value -11.6 obtained independently from acoustic harmonic generation measurements for the material. The general agreement once again affirms the role of the local energy density in the generation of static pulses.

\section{CONCLUSION}

The analytical model previously developed for acoustic rectification in nondispersive media $[1,2]$ has been extended to the case of dispersive media. The extension is obtained by assuming that the energy per unit mass $A_{\mathrm{D}}{ }^{2}$ of the rectified waves is no longer constant, as in the case of non-dispersive media, but now depends on phase factors similar to that obtained for the Earnshaw particle velocity solution to the nonlinear wave equation. We assume that at $a=0$ (the spatial position at which the toneburst is launched 
into the material) $A_{\mathrm{D}}{ }^{2}=A^{2}$ (the energy per unit mass of the toneburst at $a=0$ ). Assuming that the dispersion relation $\omega\left(k, A_{\mathrm{D}}^{2}\right)$ is a nonlinear function both of the wave number $k$ and the rectified wave amplitude $A_{\mathrm{D}}$, we expand $\omega\left(k, A_{\mathrm{D}}{ }^{2}\right)$ in a power series and use the geometrical optics postulate to obtain a Boussinesq type of equation. We add a dispersive term to the equation and solve for the energy per unit mass waveform after invoking the energy conservation equation.

The solution predicts the generation of an energy per unit mass solitary waveform (soliton). According to the analytical model the static strain pulse profile is defined locally by the energy density of the toneburst and thus has the shape of the energy density, hence energy per unit mass, profile. The static displacement pulse profile is obtained by integrating over the spatial extent of the static strain profile to yield a profile that has been observed experimentally. The agreement between the pulse profile observed experimentally and the profile predicted by the model lends validity to the assertion that the acoustic radiation-induced static pulses are dependent locally on the energy density of the propagating waveform.

\section{REFERENCES}

1. J. H. Cantrell, Phys. Rev. B 30, 3214-3220 (1984).

2. J. H. Cantrell, J. Phys. A 26, L673-L677 (1993).

3. W. T. Yost and J. H. Cantrell, Phys. Rev. B 30, 3221-3227 (1984).

4. J. H. Cantrell, W. T. Yost, and P. Li, Phys. Rev. B 35, 9780-9782 (1987).

5. S. Earnshaw, Philos. Trans. R. Soc. London 150, 133 (1860).

6. T.Taniuti and N. Yajima, J. Math. Phys. 10, 1369-1372 (1969).

7. V. I. Karpman, Nonlinear Waves in Dispervive Media, Pergamon, Oxford, 1974. 